\title{
Estimación de la degradación por mismatch del rechazo de imagen en filtros polifásicos pasivos
}

\author{
A. D. Martínez Pérez, F. Aznar, G. Royo, C. Sánchez Azqueta, S. Celma \\ Grupo de Diseño Electrónico (GDE) \\ Instituto de Investigación en Ingeniería de Aragón (I3A) \\ Universidad de Zaragoza, Mariano Esquillor s/n, 50018, Zaragoza, Spain. \\ Tel. +34-976762707, e-mail: adimar@unizar.es \\ Universidad de Zaragoza
}

\section{Resumen}

Los filtros polifásicos pasivos (PPF) son sistemas empleados extensivamente en transmisores 0 receptores heterodinos de banda lateral única (Single Side Band, SSB) para rechazar la banda imagen. Este trabajo se centra en establecer una cota a la máxima degradación que el mismatch entre componentes puede producir en su respuesta.

\section{Introducción}

El funcionamiento de los filtros polifásicos pasivos (PPF) se basa en la simetria de la red de condensadores y resistencias que los forma para realizar una conversión de señal en cuadratura a diferencial o viceversa [1]. Una señal en cuadratura está compuesta por dos componentes: fase (I) y cuadratura $(\mathrm{Q})$. Idealmente ambas son idénticas en amplitud, pero se encuentran desfasadas $90^{\circ}$ entre sí.

La cuadratura es aprovechada para cancelar la imagen, de modo que cuanto mayor sea la calidad de dicha conversión, mayor será el ratio de rechazo de imagen (Image Rejection Ratio, IRR) [2].

A causa de esto, en estándares que requieren un alto IRR (como WiFi [3]), los PPF se vuelven componentes críticos, y no-idealidades en ellos, como el mismatch, pueden provocar un desequilibrio en la red que impida alcanzar el IRR deseado [4].

\section{Análisis del mismatch}

Para estudiar el efecto que produce el mismatch en el comportamiento del filtro, se ha estudiado el caso de PPF de una etapa en circuito abierto, tal y como se muestra en la Figura 1. Desarrollando las expresiones obtenidas de dicho esquemático se ha calculado el IRR para todo el rango frecuencial (Figura 2), observándose que el error de fase $(\Delta \theta)$ es dominante en la región próxima a la frecuencia a la que está sintonizada la red, mientras que en el balance de amplitudes (ABAL) lo es en el resto del espectro. Las expresiones de dichas curvas son:

$$
\begin{gathered}
I R R_{A_{B A L}}=\left(\frac{1+\frac{1}{b(1-x)} \sqrt{\frac{1+b^{2}(1-x)^{2}}{1+b^{2}(1+x)^{2}}}}{1-\frac{1}{b(1-x)} \sqrt{\frac{1+b^{2}(1-x)^{2}}{1+b^{2}(1+x)^{2}}}}\right)^{2} \\
I R R_{\Delta \theta}=\frac{1+\frac{1+b^{2}\left(1-x^{2}\right)}{1-\frac{1+b^{2}\left(1-x^{2}\right)}{\sqrt{\left(1+b^{2}(1-x)^{2}\right)\left(1+b^{2}(1+x)^{2}\right)}}}}{\sqrt{\left(1+b^{2}(1-x)^{2}\right)\left(1+b^{2}(1+x)^{2}\right)}}
\end{gathered}
$$

siendo x la tolerancia de los componentes y b la frecuencia normalizada, definida como $b=w / w o$.

Además, a partir de dichas expresiones se puede deducir que el máximo se encontrará en el polo de IRRABal (bmax) y que el valor en dicho punto dependenderá exclusivamente del error de fase. Esto es:

$$
\begin{gathered}
1=\frac{1}{b_{\max }(1-x)} \sqrt{\frac{1+b_{\max }^{2}(1-x)^{2}}{1+b_{\max }^{2}(1+x)^{2}}} \Rightarrow \\
\Rightarrow b_{\max }=\frac{1}{\sqrt{1-x^{2}}} \\
{I R R_{\Delta \theta}\left(b_{\max }\right)}^{2}=\frac{1+\sqrt{1-x^{2}}}{1+\sqrt{1-x^{2}}} \approx \frac{4}{x^{2}}
\end{gathered}
$$

\section{Peor combinación de mismatch}

Si se considera que cada elemento puede variar en positivo $(+x)$, negativo $(-x)$ o no variar, se obtiene un conjunto finito de $3^{\mathrm{K}}$ posibilidades, siendo $\mathrm{K}$ el número de elementos de mismatch. Asimismo, es posible construir una base para el conjunto de posibilidades mediante $\mathrm{K}$ vectores $\mathrm{K}$-dimensionales linearmente independientes. 
Simulando las combinaciones que representa cada uno de los vectores de la base, es posible obtener la degradación que produce en el IRR cada uno de ellos. Además, puede deducirse que el peor caso no tendrá proyección sobre aquellos vectores de la base que no supongan una degradación significativa al IRR. Esto permite acotar sustancialmente el peor caso con una elección adecuada de las bases.

Siguiendo este método sistemático, se ha obtenido que una buena aproximación al peor caso posible es aplicar desviaciones opuestas a las ramas I y Q de la última etapa del filtro. En la Figura 3 se muestra la cota obtenida frente a un análisis de Monte Carlo de 1000 simulaciones.

\section{Conclusiones}

En este trabajo se analiza el efecto del mismatch sobre la respuesta frecuencial de los PPF. La estimación obtenida resulta una herramienta muy interesante para un diseñador de este tipo de filtros, ya que permite anticipar la tolerancia de los componentes y comprobar la validez del diseño con una única simulación, en lugar de ser necesario ejecutar centenares en un análisis de Monte Carlo.

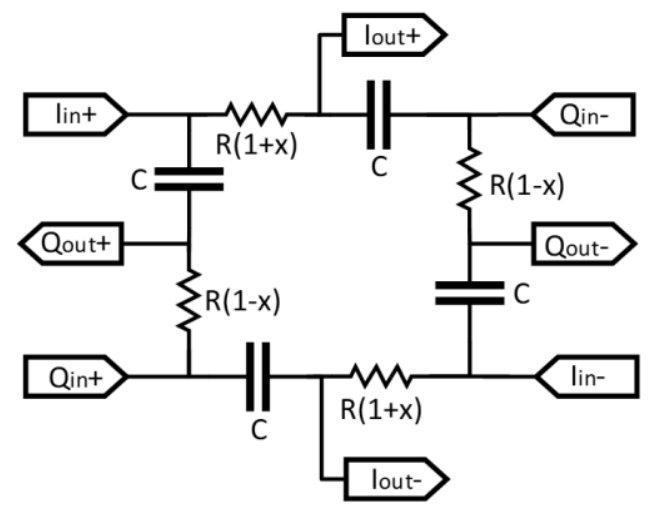

Figura 1. Esquemático de un PPF de una etapa sufriendo la peor combinación de mismatch.

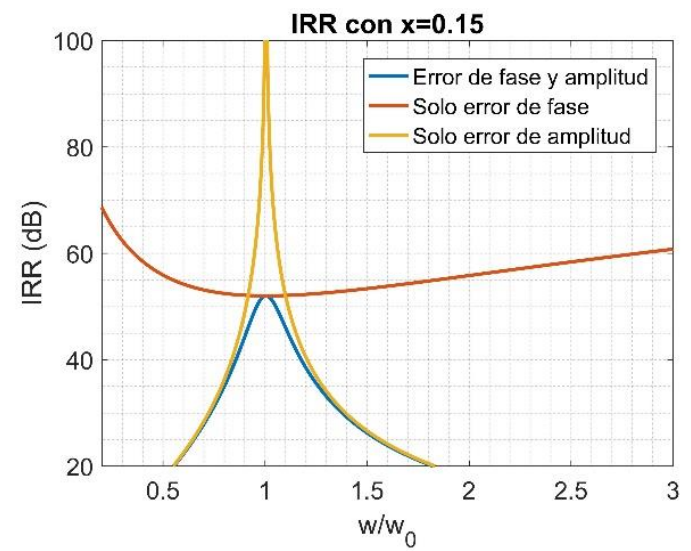

Es también de especial interés para la implementación física (layout) de este tipo de sistemas, el hecho de que el mismatch en la última etapa es crítico, por lo que deberá tener prioridad.

\section{REFERENCIAS}

[1]. BEHBAHANI, F., KISHIGAMI, Y., LEETE, J., and ABIDI, A.A. CMOS Mixers and Polyphase Filters for Large Image Rejection. IEEE Journal of Soild-State Circuits, vol. 36, no 6, 2001, pp.873-887

[2]. MARTÍNEZ-PÉREZ, A.D., AZNAR, F., ROYO, G. SÁNCHEZ-AZQUETA, C., and CELMA, S. Analysis of the Influence of Component Mismatch on Integrated Passive Polyphase Filters. IEEE International Symposium on Circuits and Systems, 2018

[3]. 802.11-2012 IEEE Std. Part 11: Wireless LAN Medium Access Control (MAC) and Physiscal Layer (PHY) Specifications

[4]. MARTÍNEZ-PÉREZ, A.D., MORTE, J., AZNAR, F., SÁNCHEZ-AZQUETA, C., and CELMA, S. Impact of Non-Idealities on Passive Polyphase Filter Performance. European Conference on Circuit Theory and Design, 2017

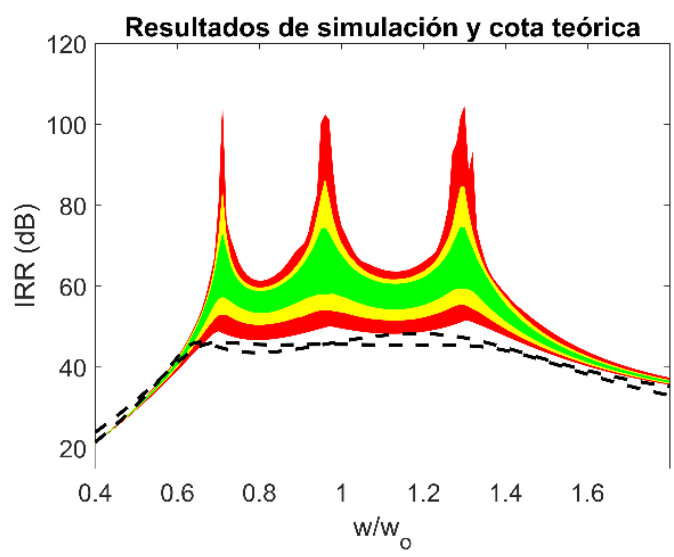

Figura 3. La cota obtenida teóricamente en negro y en color los resultados de un análisis de Monte Carlo con 1000 simulaciones. La región verde comprende el $70 \%$ de los casos; cada una de las zonas amarillas supone un 12,5\%; y entre ambas áreas rojas se recoge el 5\% más extremo de casos.

Figura 2. IRR en función de los errores de fase y amplitud

Revista "Jornada de Jóvenes Investigadores del I3A", vol. 6 (Actas de la VII Jornada de Jóvenes Investigadores del I3A - 8 de junio de 2018). ISSN 2341-4790. 